One of the urgent problems in the dairy industry is to reduce power input in the process of dispersing milk fat while ensuring a high degree of homogenization. This problem can be solved through the development and implementation of a virtually unexplored jet-slot milk homogenizer. The principle of its action implies the preliminary separation of cream from whole milk and its feed into the high-velocity flow of skim milk. The homogenization process occurs by creating a sufficient difference in velocities of the disperse and dispersing phases of the milk emulsion, which is mathematically described by Weber's criterion.

Experimental studies of the effect of fat content in cream, cream feed rate, and width of the annular slot on dispersion indices during processing in the designed homogenizer have been carried out. The mathematical dependence which relates these parameters was found. It was proved that to obtain a milk emulsion with a dispersion level of $0.8 \mu \mathrm{m}$, the width of the annular slot should be 0.1-0.5 mm, fat content in cream 40-50\%, and the feed rate less than $40 \mathrm{~m} / \mathrm{s}$. The results of the evaluation of dispersion quality show a $7 \%$ decrease in the average diameter of the fat globules compared to the most common values obtainable in the valve homogenizer. A refined critical value of the Weber criterion for dispersion of the fat phase of milk was determined (29 units) which indicates an increase in the intensity of the homogenization process in comparison with the jet milk homogenizer with a separate cream feed. The derived critical value of the criterion is necessary to create a theory of the process of dispersing milk fat and develop more efficient designs of milk homogenizers

Keywords: homogenization, jetslot homogenizer, dispersion, emulsion dispersion, Weber criterion, fat globule
UDC 637.134

DOI: $10.15587 / 1729-4061.2020 .213236$

\section{DETERMINING THE QUALITY OF MILK FAT DISPERSION IN A JET-SLOT MILK HOMOGENIZER}

\author{
K. S a mo i c h u k \\ Doctor of Technical Sciences, Associate Professor* \\ E-mail: samoichuk.k@ukr.net \\ A. Kovalyov \\ Assistant* \\ E-mail: koba_o@ukr.net \\ V. Oleksi ienko \\ $\mathrm{PhD}$, Associate Professor* \\ E-mail: vadym.oleksiienko@tsatu.edu.ua
}

N. Palia n y c h k a

$\mathrm{PhD}$, Associate Professor* E-mail: palyanichkan@gmail.com

D. D m y trevsk y i

$\mathrm{PhD}$, Associate Professor** E-mail: dmitrevskyidv@gmail.com

v. Chervony i

$\mathrm{PhD}$, Associate Professor**

E-mail: chervonyi.v@gmail.com

D. Horielkov

$\mathrm{PhD}$, Associate Professor** E-mail: gorelkov.dmv@gmail.com

I. Zolot ukhina

$\mathrm{PhD}$, Associate Professor**

E-mail: zolotce5@gmail.com

A. S I ashcheva

$\mathrm{PhD}$, Associate Professor

Department of Technologies in Restaurant Industry, Hotel and Restaurant Business and Entrepreneurship Donetsk National University of Economics and Trade named after Mikhail Tugan-Baranovsky

Tramvayna str., 16, Kryvyi Rih, Ukraine, 50005 E-mail: slashcheva@donnuet.edu.ua

*Department of Processing and Food Production Equipment named after professor F. Yalpachik

Dmytro Motornyi Tavria State Agrotechnological University Bohdana Khmelnytskoho ave., 18, Melitopol, Ukraine, 72312 **Department of Food and Hotel Industry Equipment named after M. I. Belyaev Kharkiv State University of Food Technology and Trade Klochkivska str., 333, Kharkiv, Ukraine, 61051

\section{Introduction}

Milk homogenization improves the taste of dairy products and extends their shelf life, so it belongs to standard operations used in the dairy industry. It is conducted in the production of most dairy products $[1,2]$. The purpose of homogenization is a dispersion (reduction) of the average diameter of fat globules (ADFG) of milk from $3-4 \mu \mathrm{m}$ in nonprocessed milk to $0.75-0.85 \mu \mathrm{m}$ in a finished product. Such values correspond to dispersion characteristics of valve 
homogenizers that are most common in the industry [1]. In addition, homogenizing provides uniform distribution of cream (disperse phase) in skim milk (dispersing medium). However, power input into the process is $6-8 \mathrm{kWh}$ per ton of homogenized milk $[1,3]$ when ensuring a high degree of dispersion with the use of valve homogenizers. Therefore, the improvement of the power efficiency of the homogenization process is an urgent task for scientists and specialists in the food industry.

Study and development of power-efficient designs of milk homogenizers are characterized by the difficulty of observing the fat globule destruction processes. This is because of their microscopic size (less than $1 \mu \mathrm{m}$ ), high milk velocities (up to $100 \mathrm{~m} / \mathrm{s}$ in valve homogenizers), and low transparency of the milk emulsion. Because of this, researchers of the homogenization process put forward more than 7 hypotheses of the milk fat dispersion (cavitation, gradient, turbulent, subcavitation, etc.) which contradict each other [1-4]. Homogenizers designed on the basis of such hypotheses either do not ensure dispersion of ADFG at a level of valve homogenizers (centrifugal, electrohydraulic, oscillatory homogenizers) [3] or need high power inputs (valve homogenizers, microfluidizers) $[1,4]$. According to recent studies, the main hydrodynamic criterion for the destruction of milk fat globules is determined by the Weber number which increases with increasing velocity of the fat globe relative to the milk plasma (velocity of the fat globule sliding) [2, 3, 5]. Therefore, the most efficient homogenizers are designed in such a way as to obtain the largest difference in the relative velocity of the fat and skimmed milk phases. Jet homogenizers belong to this group. In addition, jet homogenizers are characterized by the low power input $(1.0-1.8 \mathrm{kWh} / \mathrm{t})$ and high reliability [6]. Therefore, the study of the process of milk fat dispersion in order to design power-efficient jet homogenizers is promising for the present-day food industry.

\section{Literature review and problem statement}

The urgency of development of jet dispersion of emulsions is evidenced by a large number of fundamentally different designs of jet milk homogenizers which were developed recently. They include counterflow-jet, impact-jet, cavitational hydrodynamic, jet homogenizers with the separate feed of the fat phase, mini-mixers, and microfluidizers [3-15]. The latter belong to the most advanced type of homogenizers that enable obtaining of a milk emulsion with the finest dispersed composition (with ADFG of $0.1 \mu \mathrm{m}$ and less) [3]. Despite this, because of high specific power input which is higher than in valve homogenizers and low productivity of industrial designs, microfluidizers have not been widely used at milk plants [4].

The results of studies presented in [7] show that it is possible to achieve a reduction in power consumption through the use of T-shaped or U-shaped mini-mixers. Skim milk is fed in them to the flow of cream moving along the central channel at a velocity above $100 \mathrm{~m} / \mathrm{s}$ through channels perpendicular to the direction of movement of the dispersing medium. It was shown that such designs are characterized by moderate power inputs $(1.5-1.7 \mathrm{kWh} /$ ton $)$ and ADFG is $1.0-1.1 \mu \mathrm{m}$ in the homogenized state [8]. A further improvement of devices of this type is limited by the need to create a high fluid flow rate. Optimization of the shape of internal channels of mini-mixers practically does not solve this prob- lem (the results are given in [7]). In addition, micromixers do not achieve an effective impact of the cream flow on the stream of skim milk [8]. This problem can be solved by using a counterflow-jet homogenizer. Its design is described in [9]. This homogenizer contains two opposite nozzles so that the fat phase is dispersed by the collision of the milk jets. The counterflow-jet homogenizer features the creation of a high velocity of the runaround of fat globules by the dispersing medium and, as a result, high values of Weber's hydrodynamic criterion. It was shown in [9] that the counterflow-jet milk homogenizer provides ADFG at a level of $0.75-0.85 \mu \mathrm{m}$ and has a specific power input of $1.2-1.5 \mathrm{kWh} / \mathrm{t}$. However, the foaming-related issues, which occur because of the destabilization of protein particles when the milk emulsion comes into contact with air, remain unresolved. An impact-jet homogenizer in which dispersion of the fatty phase occurs during the collision of milk flow with hard walls of various shapes has similar problems. This type of homogenizer is less power-efficient than the counterflow-jet homogenizer which has caused the suspension of its further study [10,11].

The development of devices with the operation principle based on the cavitational hypothesis of dispersion of the fat phase of the milk emulsion may be an option of power efficiency improvement. This approach is used in [12,13], which provide a description of dispersers, in which cavitation is created by oscillations of plates or other working bodies due to the milk jet (flow). It was shown that such homogenizers have a simple and reliable design. However, recent studies of the cavitation effect on the destruction of milk fat globules have shown that this process is not a determining factor in homogenization $[1,14]$. These results have called into question the development of designs in which cavitation is the main driving force of the milk fat dispersion.

Separate homogenization of the fat phase is an effective way to reduce power input for dispersion. The principle of operation of a jet milk homogenizer with a separate feed of cream is given in [15]. In such a device, milk is separated before processing and then fed into the homogenizer in two separate streams: cream is fed through thin channels into the high-velocity flow of skim milk. Due to this, high values of the velocity of fat globules (Weber's criterion) are ensured which leads to a reduction of the ADFG to $0.8-0.9 \mu \mathrm{m}$ and specific power input to $0.9 \mathrm{kWh} / \mathrm{t}$ [16]. The main disadvantage of this homogenizer is the need to use channels with an inner diameter of $0.6-0.9 \mathrm{~mm}$ which results in channel obliteration and hence reduced operation reliability.

Options of overcoming the problem of low energy efficiency of dispersion include the use of counterflow-jet and jet homogenizers with a separate feed of the fat phase. However, these homogenizers have a number of disadvantages which include:

- a feed of the disperse phase through narrow channels which reduces the homogenizer reliability;

- contact of emulsion with air deteriorates the quality of the homogenized emulsion.

These shortcomings were eliminated in a jet-slot milk homogenizer with separate cream feed (JSHSCF). The device combines advantages of separate homogenization, creation of a maximum value of the Weber criterion, and the possibility of milk normalization by fat content, simultaneously with homogenization [6]. According to the results of analytical studies with the use of JSHSCF, it is possible to obtain fat globules with an average diameter of $0.8-0.9 \mu \mathrm{m}$. In this case, the specific power input of the homogenizer will not exceed $0.7-0.8 \mathrm{kWh} / \mathrm{t}$ of homogenized 
emulsion [16]. Influence of confuser shape and diameter at the point of greatest narrowing on ADFG of milk emulsion after homogenization was determined in [17]. However, the analytical study of the influence of such factors as fat content in cream, the velocity of its feed, and the width of the annular slot on dispersion quality causes difficulties because of the complex interaction of multiphase jets of emulsions in the jet homogenizer. Such problems are usually solved by computer simulation methods. However, because of the lack of a unified theory of homogenization, computer models describing the dispersion of emulsions have not yet been developed. This fact suggests that it is advisable to conduct studies to determine the impact of technological, hydraulic, and design parameters on the quality of dispersion in JSHSCF.

When studying the dispersion of fat globules (droplets) in the dispersion medium, it is important to take into account the resistance of the globule to the action of forces tending to destroy it. Their ratio is determined by Weber's criterion We $[2,18]$. When We exceeds some critical value $\mathrm{We}_{k}$ which depends on the properties of dispersing and dispersing media, destruction of the fat droplet occurs. Values of critical Weber criterion in various literature sources are in a range from 8 to 28. This is explained by the complexity of calculation or experimental establishment of the relative velocity of dispersing and disperse phases $[3,5]$. Given such a wide range of fluctuation of $\mathrm{We}_{k}$ values, refinement of this value enough for the destruction of the milk fat globules is an important task in the study of the mechanisms of dispersion of the disperse phase of the milk emulsion.

\section{The aim and objectives of the study}

The study objective consists in determining the influence of fat phase dispersion in JSHSCF on milk quality characteristics and the Weber criterion. This will make it possible to substantiate rational indicators of JSHSCF and develop an industrial design of a homogenizer for its implementation in production to reduce power input for milk homogenization.

To achieve this objective, the following tasks were set:

- to determine the effect of fat content, the width of the annular slot at the point of greatest chamber narrowing, and cream feed rate on the ADFG after dispersion in JSHSCF;

- to evaluate dispersion characteristics of the milk emulsion obtained after processing in JSHSCF;

- to determine the critical value of the Weber criterion in the homogenization of milk in JSHSCF.

\section{The procedure of milk homogenization in a jet-slot} homogenizer

4.1. Scheme and principle of the homogenizer operation

The chamber of the jet-slot milk homogenizer (Fig. 1) consists of a confuser 2 and a diffuser 5 , pipes 1 and 6 for feed- ing the starting milk and discharging the finished product, a cream container 4 with an annular slot 3 [6]. Pre-skimmed milk is fed through the nozzle 1 with a velocity $v_{s k}$. At the point of the greatest narrowing of the confuser 2 with a diameter $d_{k}$, cream is fed with velocity $v_{c}$ from the cream container through a narrow annular slot 3 with width $h$. When the annular stream of cream and the jet of skim milk enter, a high velocity of streamlining the fat globules is created and, as a consequence, the Weber criterion. As a result, the destruction of fat globules occurs during the transition to the diffuser 5 expansion. Homogenized milk is discharged through pipe 6 .

\section{Cream feed}

$\mathrm{F}_{\mathrm{c}}$

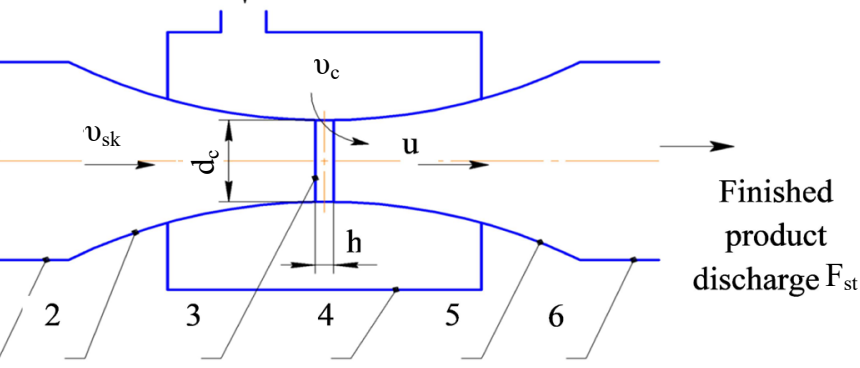

Fig. 1. Scheme of jet-slot milk homogenizer with the separate feed of cream: of skim milk feed; 2 - confuser; 3 - annular slot; 4 - container with the annular slot; $u$ is sliding city of fat globules relative to milk plasma; $F_{s k}$ is the fat content in skim milk $F_{c}$ is the fat content in cream; $F_{s t}$ is the fat content in the finished product

The developed device normalizes milk by fat content simultaneously with homogenization. Therefore, the feed rate of skim milk, cream, and fat content in cream are calculated from the ratio found in analytical studies to obtain milk with the required fat content [17].

\section{2. Methods of experimental studies}

To conduct experimental studies, a laboratory JSHSCF facility was built at the Department of Processing and Food Production Equipment named after professor F. Yalpachik at the Dmytro Motornyi Tavria State Agrotechnological University, Ukraine. The facility consists of a container 8 with skim milk coming to a gear pump 2 of NSh type via flexible hose 1 . The pump is driven by a three-phase electric motor 5 which is started when a rotary switch 4 is on.

The working pressure of skim milk is regulated by closing the throttle valve 11 and controlled by the adjacent manometer 12. After preliminary separation, the skim milk coming from the pump 2 through the pipes 6 is fed to the homogenization chamber 9 . At the narrowest point of the chamber, cream is fed to skim milk from the cream container 3 through the flexible hose 10 by means of the pump 7 . The cream feed pump is actuated when power supply 16 is connected to the mains and the toggle switch 15 is on. The required amount of cream is provided by a frequency converter 13 with a regulator. Normalized and homogenized milk is discharged through a flexible hose 14 into a special container [16]. 


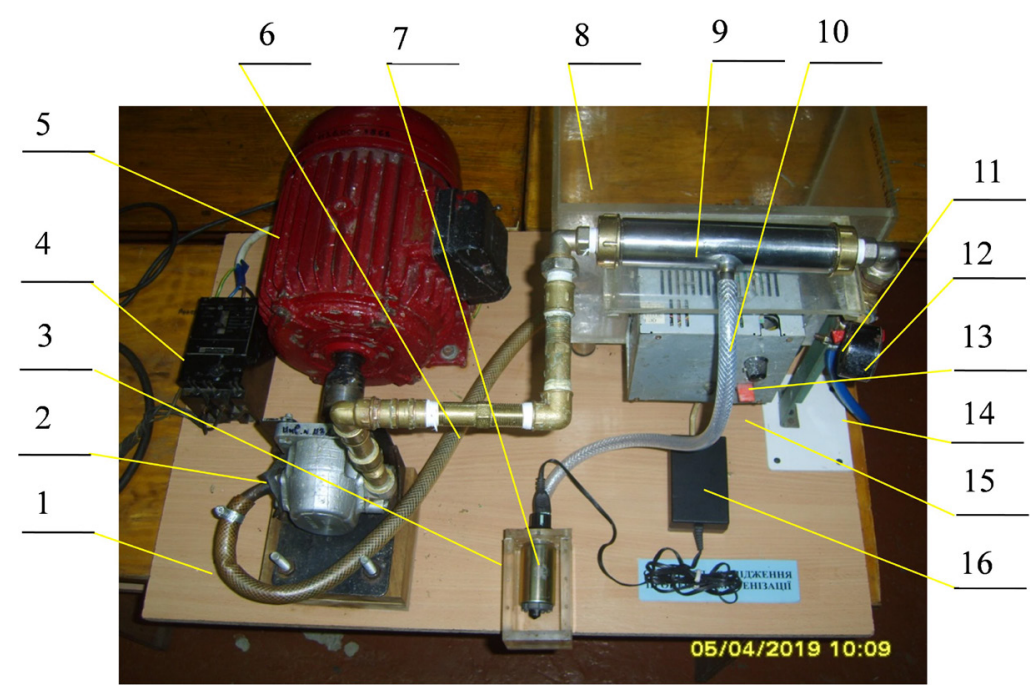

Fig. 2. Laboratory facility of a jet-slot milk homogenizer: 1 - flexible pipe for the feed of skim milk; 2 - gear pump of NSh type; 3 - container with cream; 4 - rotary switch; 5 - three-phase electric motor; 6 - pipeline;

7 - pump for feeding the disperse phase; 8 - container with skim milk; 9 - homogenization chamber; $10-$ a flexible hose with a clamp for feeding cream; 11 - throttle valve; 12 - manometer; 13 - frequency converter with regulator; 14 - flexible hose for draining homogenized milk; 15 - switch to start the cream feed pump; 16 - electric drive of the cream feed pump

Cow's milk meeting requirements ISO 9622:2013 Milk and Liquid Milk Products was used for experimental studies [19]. Sampling was performed according to ISO 707:2013 Milk and Milk Products. Guidance on Sampling [20].

Disperse composition of fat globules was determined after dispersion using Micromed R-1-LED optical microscope, Russian Federation, with a total magnification of 1,500 times. Mustek Wcam 300 digital camera, Taiwan, with $640 \times 480$ resolution [21] was connected to the microscope. Each of the experiments was repeated three times.

The temperature of skim milk and cream was the constant factor of the dispersion process in the jet-slot milk homogenizer. The optimal temperature was set at $60-$ $65^{\circ} \mathrm{C}[2,15,22]$. Fat content in cream, cream velocity, and width of the annular slot were variable factors. The following limits of variation of variable factors were established for the experiment [16]:

- fat content in cream $(10-50 \%)$;

- velocity of cream $(5-110 \mathrm{~m} / \mathrm{s})$;

- width of the annular slot $(0.1-0.9 \mathrm{~mm})$.

The quality of the milk homogenization process was determined by the ADFG parameters and the width of the distribution of the disperse composition of the milk emulsion. The latter indicator can be visually assessed by the histogram of the distribution of fat globules by size and quantitatively by the coefficient of variation.

\section{3. The procedure for determining the critical value} of the Weber criterion

The Weber criterion is the main criterion of the fat globule destruction. Its value depends mainly on the sliding velocity which is the difference between relative velocities of the dispersing and disperse phases of milk (Fig. 1) [2, 5, 11]. Average diameter $d_{a v}$ of fat globules after dispersion for jetslot homogenization depending on $\mathrm{We}_{\mathrm{k}}$ can be determined as in [3]:

$$
d_{a v}=\frac{W e_{k} \sigma_{f-p}}{2 \rho_{p} u^{2}},
$$

where $\sigma_{f-p}$ is surface tension at the phase interface "milk fat-skim milk plasma", $\mathrm{N} / \mathrm{m} ; \rho_{p}$ is the density of milk plasma, $\mathrm{kg} / \mathrm{m}^{3} ; u$ is the velocity of sliding of the fat globule relative to the milk plasma, $\mathrm{m} / \mathrm{s}$.

The velocity of sliding of the fat globule for the jet-slot milk homogenizer can be defined as a difference between velocities $v_{s k}$ and $v_{c}$ of skim milk flow and cream flow, respectively. To create the best conditions for the destruction of fat globules, the skim milk velocity should be increased. Such conditions are created in the central part of the axial line of the inner surfaces of the confuser at the slot location. At perpendicular feed of the flow of a fat phase (Fig. 1) in relation to the direction of movement of skimmed milk, the velocity of the sliding fat globule will be equal to the velocity of the flow of skimmed milk: $u=v_{s k}$. The feed of cream with a minimum velocity $v_{c}$ through an annular slot with width $\mathrm{h}$ is the condition for the adequacy of the above equality [17]. The above expression is valid only if a single fat globule is fed through the slot. In a jet homogenizer of a slot type, cream (an emulsion of fat phase in milk plasma) is fed through the slot, so the value of velocity $u$ depends on the slot width at the cream feed point, fat content in cream, and velocity of the cream flow [18, 23]:

$$
u=k_{s} v_{s k},
$$

where $k_{s}$ is the coefficient of slot homogenization with a transverse feed of the fat phase which takes into account the influence of fat content, velocity, and width of the annular slot for cream feeding.

The coefficient $k_{s}$ takes into account [23] the following:

- fat content $F_{c}$ in the cream fed to the homogenizer;

- width $h$ of the slot at the point of cream feed to the flow;

- velocity $v_{c}$ of the cream at the point of the globule transition from the slot to the skim milk flow.

Equation (2) has a coefficient that takes into account the influence of the actual flow of cream (a certain fat content, feed velocity, and the width determined by the width of the annular slot of the homogenizer chamber) on the velocity of streamlining the fat globule surface. Assuming conditions under which only a single fat globule is fed through the annular slot, in this case:

$$
-F_{c}=100 \% \text {; }
$$

$-h=d_{a v}$, that is, only one fat globule of milk passes through the annular slot;

$-v_{c}=0$, that is, the velocity of streamlining the fat globule is equal to the velocity of the plasma flow, then $k_{s}$ in equation (2) will be equal to 1 and the sliding velocity of the fat globule will be equal to the skim milk velocity.

The dependence $k_{s}=f\left(F_{c}, h, v_{c}\right)$ must be determined experimentally.

To determine the critical value of the Weber criterion, graphical dependences of ADFG on variable factors were built on the basis of the obtained data. After that, values of cream feed rate, slot width, and fat content in cream for the conditions that imitate entry of a single fat globule 
into the skim milk flow were assessed employing Microsoft Office Excel 2010.

The critical value of the Weber criterion was calculated from the following formula obtained from formula (1):

$$
W e_{k} \leq \frac{2 \rho_{p}\left(k_{s} v_{s k}\right)^{2} d_{a v}}{\sigma_{f-p}}
$$

In this formula, the values of $k_{s}, v_{s k}$, and $d_{a v}$ obtained in the experimental studies were used.

5. The results obtained in experimental studies of milk homogenization in a jet-slot homogenizer

5. 1. The effect of velocity, fat content in cream, and width of the annular slot on dispersibility of the milk emulsion

In accordance with the developed method, experimental studies of the effect of velocity, fat content in cream, and width of the annular slot on the ADFG of milk after its homogenization in JSHSCF were carried out.

The reduction of ADFG to the level obtainable in valve homogenizers can be achieved by increasing the fat content and velocity of cream feed (Fig. 3). ADFG after the dispersion at the level of dispersion in valve homogenizers was achieved at a cream feed rate $v_{c}=5-40 \mathrm{~m} / \mathrm{s}$ and at a velocity of more than $100 \mathrm{~m} / \mathrm{s}$. This is explained by the fact that in the range of values of the cream feed rate $v_{c}=40-100 \mathrm{~m} / \mathrm{s}$, a minimum difference between velocities of dispersing and disperse phases of the emulsion was ensured. It is possible to obtain high dispersibility of the product by the use of the mode which enables feed of cream at a velocity $v_{c} \geq 100 \mathrm{~m} / \mathrm{s}$. However, power input in the creation of a high-velocity flow will be high and the power efficiency of homogenization in JSHSCF will decrease. Therefore, the feed of cream at a velocity of less than $40 \mathrm{~m} / \mathrm{s}$ is the most efficient in terms of homogenization quality and power input.

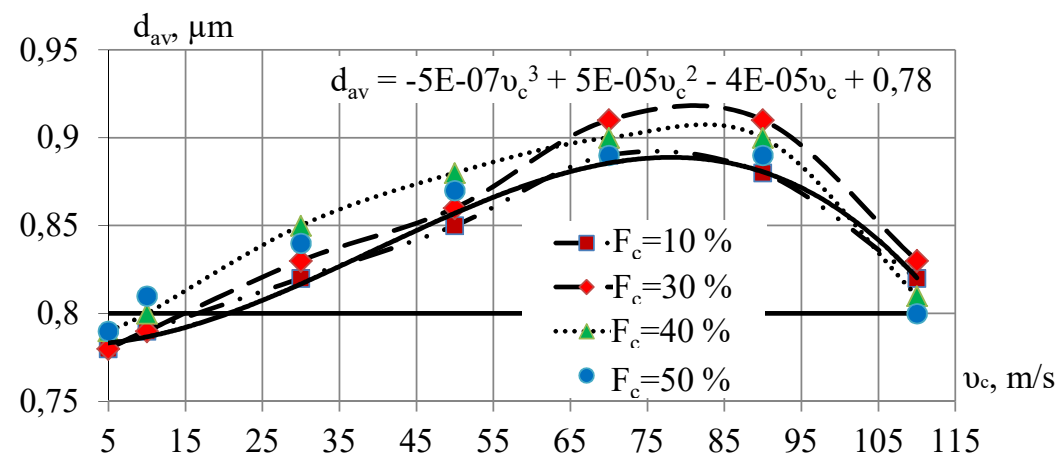

Fig. 3. Graph of the dependence of the average diameter of fat globules, $d_{a v}$, on cream velocity $v_{c}$ and fat content in cream, $F_{c}$, at h=0.5 mm, $F_{s t}=3.5 \%$, $d_{c}=3 \mathrm{~mm}, v_{s k}=60 \mathrm{~m} / \mathrm{s}$
The reduction of $\mathrm{ADFG}$ can be achieved by reducing the width of the annular slot $h$ (Fig. 4). When using a slot width of $0.1-0.5 \mathrm{~mm}$, it is possible to obtain a product with $\mathrm{ADFG}=0.75-0.85 \mu \mathrm{m}$ which is at the level of dispersibility with the use of the valve homogenizer [1-3].

As fat content in the cream fed to the JSHSCF increases, the degree of dispersion increases. The lowest values of ADFG can be achieved with the use of cream with a fat content of $40-50 \%$ (Fig. 5).

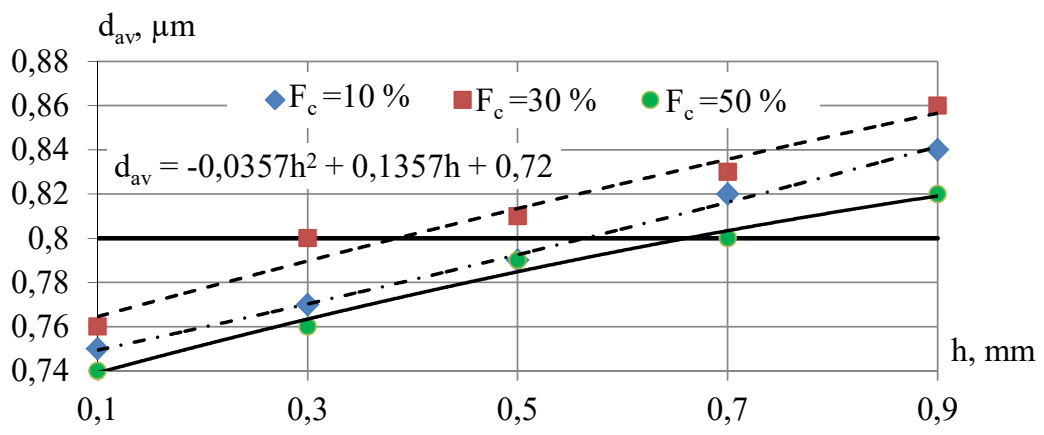

Fig. 4. Graph of the dependence of the average diameter of the fat globules, $d_{a v}$, on width of the slot of the homogenization chamber at the point of the greatest confuser narrowing, $h$, and fat content $F_{c}$ in cream at $F_{s}=3.5 \%$, $d_{c}=3 \mathrm{~mm}, v_{s k}=60 \mathrm{~m} / \mathrm{s}$

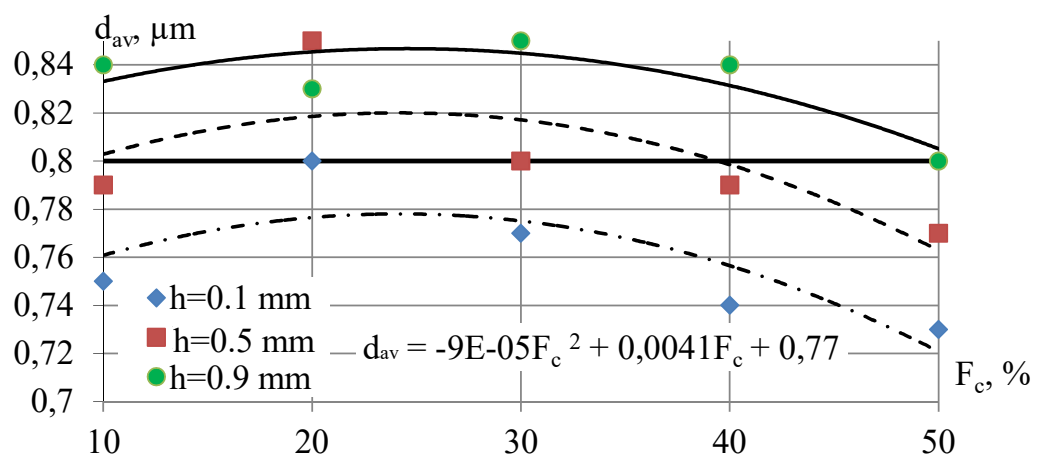

Fig. 5. Graph of the dependence of the average diameter $d_{a v}$ of fat globules on fat content $F_{c}$ in cream and width of the homogenization chamber slot at the point of the greatest narrowing $h$ at $F_{s t}=3.5 \%, d_{k}=3 \mathrm{~mm}, v_{s k}=60 \mathrm{~m} / \mathrm{s}$

With a decrease in fat content in cream to less than $10-20 \%$, a slight decrease in ADFG takes place as well. But when using the simultaneous normalization of milk by fat content, the use of cream with such fat content is practically irrational. ADFG depends on the width of the annular slot and when it increases from $h=0.1 \mathrm{~mm}$ to $h=0.9 \mathrm{~mm}$ when using cream with a fat content of $30 \%$, the dispersibility indicators deteriorate by $10 \%$.

Therefore, in order to obtain homogenized milk with a fat content of $2-4 \%$ in which ADFG is at a level of that obtainable in valve homogenizers, cream feed rate must be less than $40 \mathrm{~m} / \mathrm{s}$. At the same time, fat content in cream should be within $40-50 \%$ and the width of the annular slot should be $0.1-0.5 \mathrm{~mm}$. 
5. 2. Determination of qualitative characteristics of milk emulsion

Fig. 6 shows photomicrographs of non-homogenized milk and milk samples processed in a jet-slot milk homogenizer with a separate feed of cream.

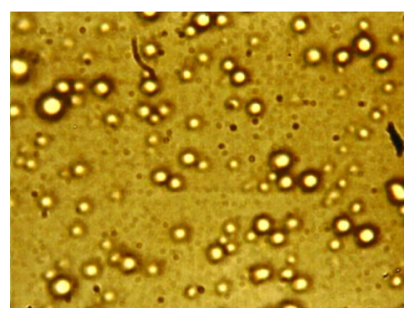

$a$

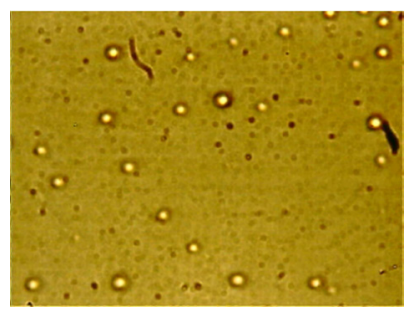

$b$

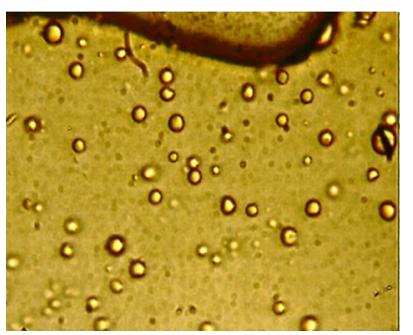

$c$

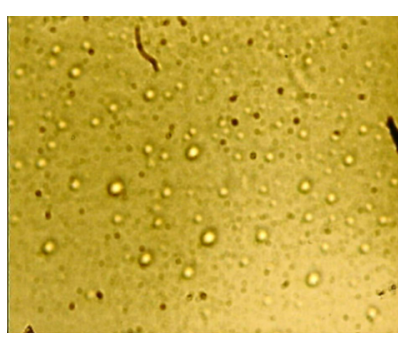

d

Fig. 6. Photomicrographs:

$a-$ unskimmed milk $\left(d_{a v}=3.2 \mu \mathrm{m}\right)$;

$b-$ milk homogenized in JSHSCF at $h=0.5 \mathrm{~mm}$;

$d_{k}=3 \mathrm{~mm} ; F_{c}=10 \%\left(d_{a v}=0.84 \mu \mathrm{m}\right) ; c-$ milk

homogenized in JSHSCF at $h=0.5 \mathrm{~mm} ; d_{k}=3 \mathrm{~mm}$; $F_{c}=30 \%\left(d_{a v}=0.91 \mu \mathrm{m}\right)$;

$d-$ milk homogenized in JSHSCF at $h=0.5 \mathrm{~mm}$; $d_{k}=3 \mathrm{~mm} ; F_{c}=40 \%\left(d_{a v}=0.79 \mu \mathrm{m}\right)$

Following the photomicrograph analysis, a histogram of ADFG distribution by size groups was constructed for the found rational parameters of fat content, cream feed rate, and slot width $\left(F_{c}=40 \%, v_{c}=20 \mathrm{~m} / \mathrm{s}\right.$, $h=0.5 \mathrm{~mm})$, Fig. 7 .

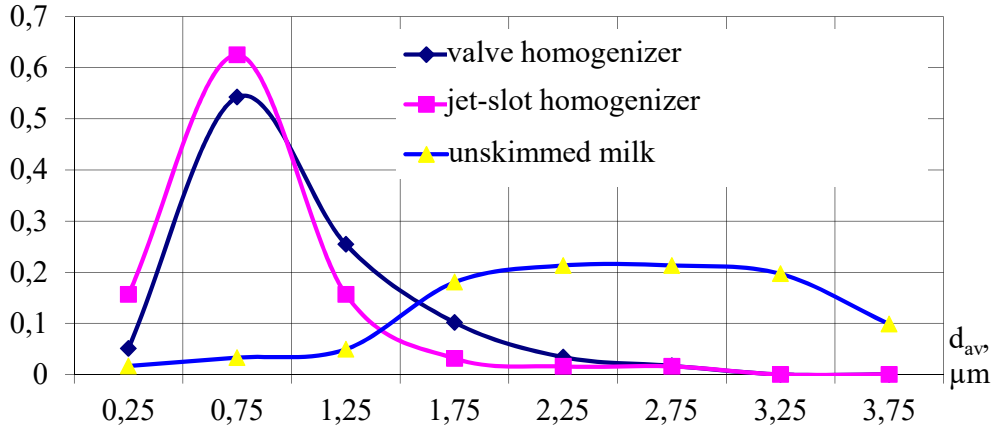

Fig. 7. Histogram of distribution of the average diameter of fat globules by size groups: $a$ - after jet-slot homogenization; $b$ - after jet homogenization with separate feed of the fat phase; $c$ - unskimmed milk

The obtained data show an increase from $55 \%$ for the jet milk homogenizer with the separate feed of cream to almost $65 \%$ for the jet-slot milk homogenizer at ADFG in the range of $0.5-1 \mu \mathrm{m}$. Milk before homogenization had the following parameters: average diameter of fat globules $d_{a v}=2.49 \mu \mathrm{m}$, dispersion $\sigma=1.66$, coefficient of variation $V=33 \%$. $\mathrm{Pa}$ rameters of milk processed in a valve homogenizer were as follows: $d_{a v}=0.85 \mu \mathrm{m}, \sigma=0.51, V=18 \%$ (at a pressure of $20 \mathrm{MPa}$ ) and when the product was processed in a jet-slot homogenizer, $d_{a v}=0.79 \mu \mathrm{m}, \sigma=0.48, V=15 \%$.

The histogram data show that after processing in JSHSCF, the coefficient of variation was $17 \%$ lower than the same indicator for the valve homogenizers. In this case, the ADFG of the product processed in JSHSCF was $7 \%$ smaller than that obtained in the valve homogenizers which are among the best in this regard [2,24].

\section{3. Determining the critical value of the Weber cri-} terion

Graphs of dependences $k_{h}=f(h), k_{f}=f(F), k_{v}=f(v)$ were constructed using the experimental data (Fig. 3-5). The experimental data were extrapolated to the conditions under which $k_{s}=1$ (Fig. 8-10).

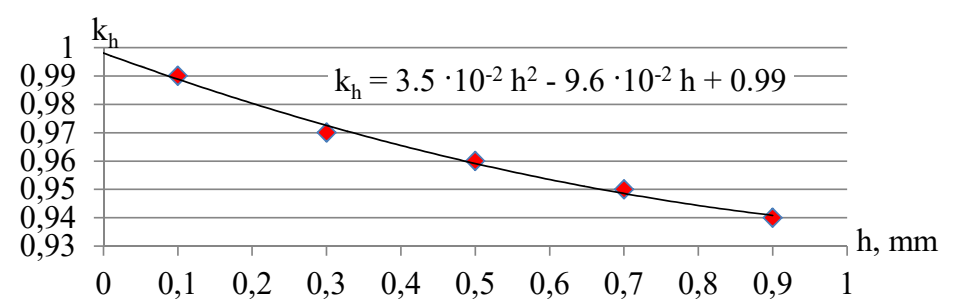

Fig. 8. Graph of the dependence of the coefficient of influence of the annular slot width $k_{h}$ on the annular slot width $\mathrm{h}$ at the point of cream feed

As a result, empirical expressions were obtained for the components of $k_{\mathrm{s}}$ :

$$
\begin{aligned}
& k_{h}=3.5 \cdot 10^{-2} h^{2}-9.6 \cdot 10^{-2} h+0.99, \\
& k_{v}=2 \cdot 10^{-5} v_{c}^{2}-2.7 \cdot 10^{-3} v_{c}+0.99, \\
& k_{f}=5 \cdot 10^{-5} F_{c}^{2}-2.2 \cdot 10^{-3} F_{c}+0.64 .
\end{aligned}
$$

Taking into account equations (4) to (6), formula (1) takes the form:

$$
d_{a v}=\frac{W e_{k} \sigma_{f-p}}{2 \rho_{p}\left[\left(3.5 \cdot 10^{-2} h^{2}-9.6 \cdot 10^{-2} h+0.99\right)\left(2 \cdot 10^{-5} v_{c}^{2}-2.7 \cdot 10^{-3} v_{c}+0.99\right)\left(5 \cdot 10^{-5} F_{c}^{2}-2.2 \cdot 10^{-3} F_{c}+0.64\right)\right]^{2} v_{s k}^{2}} .
$$




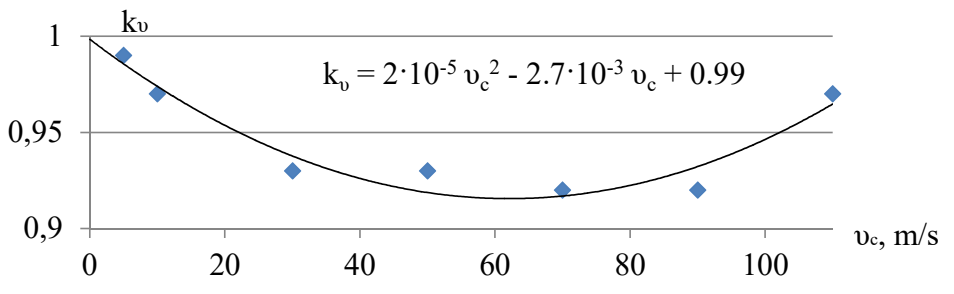

Fig. 9. Graph of the dependence of the coefficient of influence of cream feed rate, $k_{v}$, on the cream feed rate, $v_{c}$

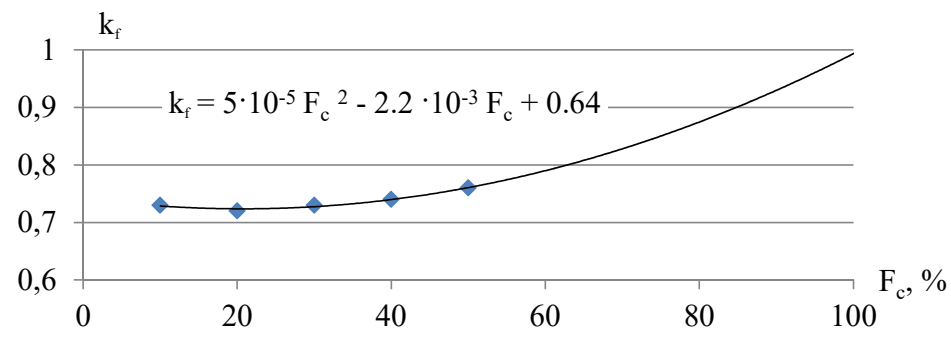

Fig. 10. Graph of the dependence of the coefficient of influence of fat content in cream, $k_{\mathrm{f}}$, on fat content in cream, $\mathrm{F}_{\mathrm{c}}$

According to formula (3), the critical value of the Weber criterion was 29 at $\rho_{p}=1,030 \mathrm{~kg} / \mathrm{m}^{3}, d_{a v}=0.75 \mu \mathrm{m}$, $v_{s k}=60 \mathrm{~m} / \mathrm{s}$ (at the confuser diameter at the point of greatest narrowing, $\left.d_{c}=3.5 \mathrm{~mm}\right), \sigma_{f-p}=0.1 \mathrm{~N} / \mathrm{m}$.

The obtained formula (7) makes it possible to choose technological, hydraulic and design parameters of JSHSCF operation to ensure the required value of ADFG of the milk emulsion at the stage of design of the JSHSCF commercial prototype.

\section{Discussion of the results obtained in the study of quality of milk emulsion dispersion}

The results obtained in the study are a continuation of the developments published in the series of articles devoted to mathematical model construction, determination of quality indicators, and power consumption of JSHSCF [16, 17, 23]. The jet-slot milk homogenizer features an ability to direct a thin stream of cream through a narrow annular slot to the rapid flow of skimmed milk. Implementation of this method makes it possible to appreciably cut (up to 6-8 times) specific power input for dispersion while ensuring dispersibility at a level provided by the valve homogenizers [16]. The study limitations include the lack of photomicrographs at the stages of the process of destruction of milk fat globules during homogenization in JSHSCF. This is explained by significant technical difficulties associated with the high velocity of the movement of fat globules and their microscopic size [24, 25].

The results of experimental studies (Fig. 3) confirm the possibility of obtaining a product with dispersion indicators at the level obtainable in valve homogenizers at a feed rate of less than $40 \mathrm{~m} / \mathrm{s}$. Such parameters are close to the results of studies of the jet milk homogenizers with the separate feed of cream in which the rational value of the cream feed rate was less than $30 \mathrm{~m} / \mathrm{s}$ [18]. The discrepancy in results is explained by the presence of several channels in the jet milk homogenizer which makes it possible to ensure the required performance of the homogenizer at a lower feed rate. An increase in
ADFG was observed in the range of cream feed rate of $40-100 \mathrm{~m} / \mathrm{s}$. This is explained by the fact that to obtain a milk emulsion with dispersibility at the level provided by valve homogenizers, the feed rate of skim milk should be $60-90 \mathrm{~m} / \mathrm{s}$ as the results of analytical studies have shown [17]. When the cream feed rate exceeded $100 \mathrm{~m} / \mathrm{s}$, a decrease in ADFG was observed. However, the application of this mode is characterized by low power efficiency. The power input to create a thin stream of viscous liquid (milk cream) at a velocity of more than $100 \mathrm{~m} / \mathrm{s}$ becomes close to the power input in the valve homogenizer $[1,2,5]$. The obtained results correlate well with the dependences found for the jet milk homogenizer with the separate feed of cream [5, 26, 27].

It is possible to obtain a milk emulsion with ADFG at the level of that obtained in valve homogenizers by reducing the width of the annular slot (Fig. 4). At the same time, the central zone of the cream jet with a reduced flow rate of fat globules becomes smaller. The reduction of this zone leads to an increase in the degree of emulsion dispersion. Thus, the rational value of the annular slot width should tend to a minimum. However, too low values of the slot width result in an increased obliteration of the inner surfaces which will reduce the reliability of the experimental homogenizer. Therefore, the rational values of the slot width are $0.1-0.5 \mathrm{~mm}$. The obtained range of values is expected to be smaller than the diameter of cream feed channels in the jet milk homogenizer with separate cream feed $(0.6-0.8 \mathrm{~mm})$ [18]. The cross-sectional area of the annular slot in JSHSCF is close to the values of the total area of the cream channels in the jet homogenizer with a separate feed of cream. However, when using an annular slot instead of tubular channels, it becomes possible to reduce the width of the slot which increases the degree of dispersion of the milk emulsion (Fig. 4).

Experimental studies have shown that the increase in dispersion of the milk emulsion occurs when the fat content in the cream increases to $50 \%$ (Fig. 5). This is because of the inefficient dissipation of energy of the skim milk jet into which the jet of cream is introduced, to the milk plasma contained in the cream. Therefore, with an increase in the amount of plasma in the cream flow (which is inversely proportional to their fat content), the rate of streamlining of the fat globules by the skim milk decreases and hence the degree of dispersion gets smaller.

Limits of fluctuation of the obtained range of rational values of fat content in cream are in good agreement with the dependences of the jet milk homogenizer with a separate cream feed [18]. Rational fat content in cream varies for it in the range of $35-45 \%$ while the ADFG fluctuation is $0.8-$ $0.9 \mu \mathrm{m}$ [18]. Studies of rational parameters of a T-shaped mini mixer show that the rational value of the cream feed rate is $40 \%[28,29]$. When using it, a milk emulsion is obtained with ADFG varying in the range of $1.0-1.1 \mu \mathrm{m}$ [30]. The use of cream with fat content more than $50 \%$ is irrational in view of a significant increase in power input for additional homogenizing the high-fat cream [31, 32].

The critical value of the Weber criterion $\mathrm{We}_{k}=29$ was experimentally determined for JSHSCF. The obtained results show its increase in comparison with the values known from other studies. For example, the critical value of the Weber criterion is $8-10$ units when dispersing in air and 28 units 
for a jet milk homogenizer with a separate cream feed [23, 5]. The difference between the density of the disperse and dispersing phases is 2 orders of magnitude less than for liquid and air. The increase in the criterion value for emulsions compared to gas occurs due to the greater involvement in the movement of adjacent layers of the liquid surrounding the globule compared with the course of dispersion in the air [26, 27]. The distinction of the obtained critical value of the Weber criterion from that obtained with the jet milk homogenizer with a separate cream feed is explained by greater involvement of adjacent layers. This effect is achieved when an annular slot is used in JSHSCF for feeding cream instead of channels. This leads to an increase in relative velocity and, as a consequence, to an increase in the intensity of the homogenization process [5, 27].

The obtained results of breaking the ADFG into size groups (Fig. 6) show a $17 \%$ decrease in the coefficient of variation and increase in dispersion in the developed homogenizer compared to the valve homogenizer $[2,33]$. This improvement in the quality of the milk emulsion correlates with values of the Weber criterion for the JSHSCF and the valve homogenizer [5]. Therefore, the obtained results confirm the hypothesis of the decisive influence of the difference between velocities of the disperse and dispersing phases on the degree of dispersion [5, 21,27].

The study limitation consists in the lack of data on the degree of obliteration of inner surfaces of the annular slot during prolonged operation of the homogenizer. This can potentially lead to lower productivity and higher specific power input. It is planned to conduct a study on the impact of obliteration after the development of a commercial JSHSCF prototype is completed.

\section{Conclusions}

1. A relationship between cream velocity, the width of the annular slot, fat content in cream, and average diameter of fat globules after dispersion in JSHSCF was established. Its analysis has allowed us to find rational parameters of the homogenizer to ensure ADFG at a level of $0.75-0.85 \mu \mathrm{m}$ : cream velocity shall be $40 \mathrm{~m} / \mathrm{s}$ and less, fat content in cream of $40-50 \%$, and the ring slot width of $0.1-0.5 \mathrm{~mm}$.

2. Dispersion characteristics of the milk emulsion obtained by its processing in the JSHSCF were evaluated. The coefficient of variation was $15 \%$ and the ADFG of milk emulsion was $0.79 \mu \mathrm{m}$ which is lower by 17 and $7 \%$, respectively, than for valve homogenizers. This proves the high quality of the milk emulsion after processing in the experimental homogenizer.

3. Experimental data based on extrapolation of experimental data have allowed us to refine the critical value of the Weber criterion for milk dispersion equal to 29 units. The value found shall be used in the creation of mathematical models of milk fat dispersion and the development of more efficient designs of milk homogenizers.

\section{References}

1. Fialkova, E. A. (2006). Gomogenizatsiya. Noviy vzglyad. Sankt-Peterburg: GIORD, 392.

2. Nuzhin, E. V., Gladushnyak, A. K. (2007). Gomogenizatsiya i gomogenizatory. Odessa: Pechatniy dom, 263.

3. Huppertz, T. (2011). Homogenization of Milk | Other Types of Homogenizer (High-Speed Mixing, Ultrasonics, Microfluidizers, Membrane Emulsification). Encyclopedia of Dairy Sciences, 761-764. doi: https://doi.org/10.1016/b978-0-12-374407-4.00226-0

4. Ciron, C. I. E., Gee, V. L., Kelly, A. L., Auty, M. A. E. (2010). Comparison of the effects of high-pressure microfluidization and conventional homogenization of milk on particle size, water retention and texture of non-fat and low-fat yoghurts. International Dairy Journal, 20 (5), 314-320. doi: https://doi.org/10.1016/j.idairyj.2009.11.018

5. Samoichuk, K., Zahorko, N., Oleksiienko, V., Petrychenko, S. (2019). Generalization of Factors of Milk Homogenization. Modern Development Paths of Agricultural Production, 191-197. doi: https://doi.org/10.1007/978-3-030-14918-5_21

6. Deinychenko, G., Samoichuk, K., Kovalyov, O. (2016). Constructions of jet mixing dispergators of milk fat phase. Proceedings of the Tavria State agrotechnological university, 1 (16), 219-227.

7. Fonte, C. P., Fletcher, D. F., Guichardon, P., Aubin, J. (2020). Simulation of micromixing in a T-mixer under laminar flow conditions. Chemical Engineering Science, 222, 115706. doi: https://doi.org/10.1016/j.ces.2020.115706

8. Roudgar, M., Brunazzi, E., Galletti, C., Mauri, R. (2012). Numerical Study of Split T-Micromixers. Chemical Engineering \& Technology, 35 (7), 1291-1299. doi: https://doi.org/10.1002/ceat.201100611

9. Samoichuk, K., Zhuravel, D., Palyanichka, N., Oleksiienko, V., Petrychenko, S., Slobodyanyuk, N. et. al. (2020). Improving the quality of milk dispersion in a counter-jet homogenizer. Potravinarstvo Slovak Journal of Food Sciences, 14, 633-640. doi: https:// doi.org/10.5219/1407

10. Jiang, B., Shi, Y., Lin, G., Kong, D., Du, J. (2019). Nanoemulsion prepared by homogenizer The CFD model research. Journal of Food Engineering, 241, 105-115. doi: https://doi.org/10.1016/j.jfoodeng.2018.08.014

11. Morales, J. O., Watts, A. B., McConville, J. T. (2016). Mechanical Particle-Size Reduction Techniques. AAPS Advances in the Pharmaceutical Sciences Series, 165-213. doi: https://doi.org/10.1007/978-3-319-42609-9_4

12. Ashokkumar, M., Bhaskaracharya, R., Kentish, S., Lee, J., Palmer, M., Zisu, B. (2009). The ultrasonic processing of dairy products An overview. Dairy Science \& Technology, 90 (2-3), 147-168. doi: https://doi.org/10.1051/dst/2009044

13. Mohammadi, V., Ghasemi-Varnamkhasti, M., Ebrahimi, R., Abbasvali, M. (2014). Ultrasonic techniques for the milk production industry. Measurement, 58, 93-102. doi: https://doi.org/10.1016/j.measurement.2014.08.022

14. Bratsikhin, A., Leschenko, E., Kostenko, K. (2019). Influence of cavitation disintegration on dairy foods production. Journal of Hygienic Engineering and Design, 27, 173-177. 
15. Samoichuk, K., Zhuravel, D., Viunyk, O., Milko, D., Bondar, A., Sukhenko, Y. et. al. (2020). Research on milk homogenization in the stream homogenizer with separate cream feeding. Potravinarstvo Slovak Journal of Food Sciences, 14, $142-148$. doi: https://doi.org/10.5219/1289

16. Samoichuk, K., Kovalyov, A., Palyanichka, N., Kolodiy, A., Lebed, M. (2019). An experimental study of parameters in the slot type jet-mixing homogenizer of milk. Proceedings of the Tavria State Agrotechnological University, 2 (19), 117-129. doi: https://doi.org/ 10.31388/2078-0877-19-2-117-129

17. Samoichuk, K., Kovalyov, A., Borokhov, I., Palyanichka, N. (2019). An analytical study of the energy characteristics and parameters of dispersion quality in the homogenizer of milk jet-slot type. Proceedings of the Tavria State agrotechnological university, $1(19), 3-18$.

18. Samoichuk, K. O., Kovalyov, A. A. (2016). The mechanizms of fat globules in jet-mixing homogenizer of milk. Naukovi pratsi ONAKhT, 80 (1), 103-107.

19. ISO 9622:2013. Milk and liquid milk products.

20. ISO 707:2013. Milk and milk products. Guidance on sampling.

21. Samoichuk, K., Kiurchev, S., Oleksiienko, V., Palyanichka, N., Verholantseva, V. (2016). Research into milk homogenization in the pulsation machine with a vibrating rotor. Eastern-European Journal of Enterprise Technologies, 6 (11 (84)), 16-21. doi: https:// doi.org/10.15587/1729-4061.2016.86974

22. Samoichuk, K., Kovalov, A, Ivzhenko, A. (2012). Analysis of methods of estimation of quality of homogenization of milk. Proceedings of the Tavria State agrotechnological university, 4 (12), 222-230.

23. Kovalov, O. O., Palianychka, N. O., Lebid, M. R. (2018). Obgruntuvannia koefitsientu strumynnoi homohenizatsiyi. Ahroekolohichni aspekty vyrobnytstva ta pererobky produktsiyi silskoho hospodarstva: materialy mizhnar. nauk.-prakt. konf. Melitopol-Kyrylivka: TDATU, 46.

24. Chapter 6.3. Homogenisers. Dairy Processing Handbook (2003). Lund, 115-122.

25. Wang, X., Wang, Y., Li, F., Li, L., Ge, X., Zhang, S., Qiu, T. (2020). Scale-up of microreactor: Effects of hydrodynamic diameter on liquid-liquid flow and mass transfer. Chemical Engineering Science, 226, 115838. doi: https://doi.org/10.1016/j.ces.2020.115838

26. Liao, Y., Lucas, D. (2009). A literature review of theoretical models for drop and bubble breakup in turbulent dispersions. Chemical Engineering Science, 64 (15), 3389-3406. doi: https://doi.org/10.1016/j.ces.2009.04.026

27. Tartar, L. (2009). The General Theory of Homogenization. Lecture Notes, 470.

28. Fani, A., Camarri, S., Salvetti, M. V. (2013). Investigation of the steady engulfment regime in a three-dimensional T-mixer. Physics of Fluids, 25 (6), 064102. doi: https://doi.org/10.1063/1.4809591

29. Stankiewicz, A., Moulijn, J. A. (2002). Process Intensification. Industrial \& Engineering Chemistry Research, 41 (8), $1920-1924$. doi: https://doi.org/10.1021/ie011025p

30. Hussong, J., Lindken, R., Pourquie, M., Westerweel, J. (2009). Numerical Study on the Flow Physics of a T-Shaped Micro Mixer. IUTAM Bookseries, 191-205. doi: https://doi.org/10.1007/978-90-481-2626-2_15

31. Stepanova, L. I. (2000). Spravochnik tehnologa molochnogo proizvodstva. Vol. 1. Tsel'nomolochnye produkty. Sankt-Peterburg: GIORD, 384.

32. Walstra, P., Geurts, T. J., Noomen, A., Jellema, A., Van Boekel, M. A. J. S. (1999). Dairy technology: Principles of Milk Properties and Processes. Part II: Processes. New York: Marcel Dekker Inc, 246.

33. Postelmans, A., Aernouts, B., Jordens, J., Van Gerven, T., Saeys, W. (2020). Milk homogenization monitoring: Fat globule size estimation from scattering spectra of milk. Innovative Food Science \& Emerging Technologies, 60, 102311. doi: https://doi.org/ 10.1016/j.ifset.2020.102311 